\title{
Taking Over the Baton
}

In a relay race, the change of runners is an exciting moment. Will the lead be saved? Will the team finally win the race?

As of March 1st, I got handed on the baton from Michael Mulvany who had served as Editor for more than 10 years. He did a phenomenal work. Together with his editorial team he gave JVR its identity and made it a well renowned journal with high scientific standards that focuses on basic research in vascular and cellular biology. In the past two years, whilst working with Michael as a Co-editor, I have had the opportunity to get an insight into his friendly and fair way of handling review processes, his extremely broad knowledge and his feel for important scientific questions in the field.

Now, a new round is about to start. I am glad that I can rely on an excellent Editorial Board and the continued support of my Co-editor Gerald Meininger. New Consulting Editors will guarantee with their expertise that the best referees will evaluate the submitted articles and that top quality articles will be published. These articles should be reviewed and published as fast as possible since science is rapidly evolving. As of July there will be a full electronic submission and review process, and electronic prepublication should help to reduce the backlog between acceptance and appearance of the papers. Regular invited reviews should help to keep the readers informed about new developments in the field of vascular biology and microcirculation.

To increase the number of scientists who have access to the journal, new ways of electronic access are being considered. One example is the fact that the members of the European Society of Microcirculation, whose official journal JVR is, have free access to the articles. The Internet Discussion Forum which always focuses on one article of the upcoming issue should also help to get an impression of the journal's scientific content.

In this new round it is still our aim to further develop JVR as a top quality European journal in the exciting and exploding field of vascular research that is attractive to the many excellent researchers in this field. Without their contributions and the expert help of many referees who spend their time, these goals cannot be reached. The new editorial team will try its very best to see that this round will become a very successful one. 